\title{
Vestibular paroxysmia: Diagnostic criteria
}

\author{
Michael Strupp ${ }^{\mathrm{a}, *}$, Jose A. Lopez-Escamez ${ }^{\mathrm{b}}, \mathrm{Ji}_{-}$Soo Kim ${ }^{\mathrm{c}}$, Dominik Straumann ${ }^{\mathrm{d}}$, Joanna C. Jen ${ }^{\mathrm{e}}$, \\ John Carey ${ }^{\mathrm{f}}$, Alexandre Bisdorff ${ }^{\mathrm{g}}$ and Thomas Brandt ${ }^{\mathrm{a}}$ \\ ${ }^{a}$ Department of Neurology and German Center for Vertigo and Balance Disorders, University Hospital Munich, \\ University of Munich, Germany \\ ${ }^{\mathrm{b}}$ Otology and Neurotology Group CTS495, Department of Genomic Medicine - Centre for Genomics and \\ Oncology Research - Pfizer/Universidad de Granada/Junta de Andalucía (GENyO), PTS, Granada \\ and Department of Otolaryngology, University Hospital Granada, Spain \\ ${ }^{\mathrm{c} D e p a r t m e n t ~ o f ~ N e u r o l o g y, ~ S e o u l ~ N a t i o n a l ~ U n i v e r s i t y ~ C o l l e g e ~ o f ~ M e d i c i n e, ~ S e o u l ~ N a t i o n a l ~ U n i v e r s i t y ~ B u n d a n g ~}$ \\ Hospital, South Korea \\ ${ }^{\mathrm{d}}$ Department of Neurology, University Hospital Zurich, University of Zurich, Switzerland \\ ${ }^{\mathrm{e}}$ Department of Neurology \& Neurobiology, University of California, Los Angeles, CA, USA \\ ${ }^{\mathrm{f}}$ Department of Otorhinolaryngology, Johns Hopkins University School of Medicine, Baltimore, USA \\ ${ }^{\mathrm{g}}$ Department of Neurology, Centre Hospitalier Emile Mayrisch, Esch, Luxemburg
}

Received 1 February 2016

Accepted 29 July 2016

\begin{abstract}
This paper describes the diagnostic criteria for vestibular paroxysmia (VP) as defined by the Classification Committee of the Bárány Society. The diagnosis of VP is mainly based on the patient history and requires: A) at least ten attacks of spontaneous spinning or non-spinning vertigo; B) duration less than 1 minute; C) stereotyped phenomenology in a particular patient; D) response to a treatment with carbamazepine/oxcarbazepine; and F) not better accounted for by another diagnosis. Probable VP is defined as follows: A) at least five attacks of spinning or non-spinning vertigo; B) duration less than 5 minutes; C) spontaneous occurrence or provoked by certain head-movements; D) stereotyped phenomenology in a particular patient;

E) not better accounted for by another diagnosis.

Ephaptic discharges in the proximal part of the 8th cranial nerve, which is covered by oligodendrocytes, are the assumed mechanism. Important differential diagnoses are Menière's disease, vestibular migraine, benign paroxysmal positional vertigo, epileptic vestibular aura, paroxysmal brainstem attacks (in multiple sclerosis or after brainstem stroke), superior canal dehiscence syndrome, perilymph fistula, transient ischemic attacks and panic attacks. Current areas of uncertainty in the diagnosis of VP are: a) MRI findings of vascular compression which are not diagnostic of the disease or predictive for the affected side because they are also observed in about $30 \%$ of healthy asymptomatic subjects; and b) response to treatment with carbamazepine/oxcarbazepine supports the diagnosis but there are so far no randomized controlled trials for treatment of VP.
\end{abstract}

Keywords: Vertigo, dizziness, attacks, neurovascular compression, ICVD

\section{Introduction}

The Bárány Society representing the international community of basic scientists, otolaryngologists and neurologists committed to vestibular research mandated a Classification Committee for an International

${ }^{*}$ Corresponding author: Michael Strupp, MD, FANA, FEAN Department of Neurology and German Center for Vertigo and Balance Disorders, University Hospital Munich, Campus Grosshadern, Marchioninistrasse 15, 81377 Munich, Germany. Tel.: +49 8944007 3678; Fax: +49 8944007 6673; E-mail: michael.strupp@med.uni-muenchen.de.
Classification of Vestibular Disorders (ICVD). Individual disorders are defined by subcommittees that include otolaryngologists and neurologists from at least three continents. Since the beginning of the process, consensus papers on the classification of vestibular symptoms [4], vestibular migraine [26], Menière's disease [28] and benign paroxysmal positional vertigo (BPPV) [42] have been published.

In 1975 Jannetta and colleagues described a "neurovascular cross-compression in patients with hyperactive dysfunction symptoms of the eighth cranial nerve" [17]. This concept was further 
evaluated - mainly by neurosurgeons - in terms of the pathophysiology, area of compression and treatment by decompression of the eighth nerve [2, 12, 16, 23, 32]. In 1984 it was called "disabling positional vertigo" by Jannetta and colleagues [18], from a clinical point of view a heterogeneous syndrome of vertigo with symptoms of various durations (from seconds to days), various characteristic features (spinning vertigo, light-headedness or gait instability without vertigo), and varying accompanying symptoms. In 1986, Jannetta and colleagues [31] reported a case series of 21 patients treated by microvascular decompression, 16 of whom benefited from this procedure. These results were confirmed by larger case series on 41 patients [29] and 207 patients [30] with a success rate between $73 \%$ and $80 \%$. Later it was criticized that the selection criteria for surgery were variable and that no standardized outcome measures were used [43].

The term "vestibular paroxysmia" (VP) was introduced by Brandt and Dieterich in 1994 [5]. The initial criteria of VP were based on an observational study on 11 patients and were as follows [5]: 1) short attacks of spinning or non-spinning vertigo lasting from seconds to minutes; 2) attacks frequently dependent on a particular head position; 3 ) hyperacusis or tinnitus (7 patients) permanently or during the attack; and 4) auditory or vestibular deficits measurable by neurophysiological methods. To make the diagnosis three out of four criteria had to be fulfilled and the patient had to respond to treatment with carbamazepine.

In 2008, based on a study on 32 patients, the suggested diagnostic criteria were modified as follows to include two categories [14]: "Definite VP": at least five attacks and the patient also fulfils criteria A-E: A) vertigo spells lasting seconds to minutes. The individual attack is self-limiting and subsides without specific therapeutic intervention; B) one or several of the following provoking factors to induce the attacks: 1 . occurring at rest; 2 . certain head/body positions (not BPPV-specific positioning maneuvers); 3. changes in head/body position (not BPPV-specific positioning maneuvers); C) none or one or several of the following characteristics during the attacks: 1. disturbance of stance; 2 . disturbance of gait; 3. unilateral tinnitus; 4. unilateral pressure/numbness in or around the ear; 5. unilaterally reduced hearing; D) one or several of the following additional diagnostic criteria: 1 . neurovascular crosscompression demonstrated on MRI (CISS sequence, TOF MR angiography); 2. hyperventilation-induced nystagmus as measured by oculography; 3 . increase of vestibular deficit at follow-up investigations as measured by oculography; 4 . treatment response to antiepileptics (not applicable at first consultation); E) the symptoms cannot be explained by another disease. The diagnostic criteria for "Probable VP" were as follows: at least five attacks and the patient fulfils criterion A, and at least three of criteria B-E.

In 2009, an analogous clinical syndrome in two patients with brief, spontaneous, recurrent attacks of tinnitus and vertigo, which responded to low-dose gabapentin, was described as "audiovestibular paroxysmia" [35].

\section{Methods}

This work forms part of an ongoing multi-year project to develop an International Classification of Vestibular Disorders (ICVD) which uses a structured process to develop international consensus definitions for vestibular symptoms, syndromes, disorders, and diseases. This process, overseen by the Classification Committee of the Bárány Society (CCBS), is based on expert, multi-disciplinary committees with international representation developing diagnostic criteria for subsequent comment and refinement prior to publication. These criteria are based on a critical appraisal of current best scientific evidence. All definitions are supported by notes, comments, and written discussion according to a template established by the CCBS for ICVD. The criteria for VP were developed iteratively over a four-year period (201316) through discussion, presentation, and refinement. Special care was taken that the criteria are practical and can be applied in every country all over the world; this applies particularly to the use of laboratory examinations not available everywhere.

\subsection{Diagnostic criteria for vestibular paroxysmia}

\subsubsection{Vestibular paroxysmia (each point needs to be fulfilled)}

A) At least ten attacks ${ }^{1}$ of spontaneous ${ }^{2}$ spinning or non-spinning vertigo ${ }^{3}$

B) Duration less than 1 minute 4

C) Stereotyped phenomenology in a particular patient $^{5,6}$

D) Response to a treatment with carbamazepine/ oxcarbazepine $^{7}$

E) Not better accounted for by another diagnosis. 


\subsubsection{Probable vestibular paroxysmia (each point needs to be fulfilled)}

A) At least five attacks ${ }^{1}$ of spinning or nonspinning vertigo 3

B) Duration less than 5 minutes ${ }^{4}$

C) Spontaneous occurrence or provoked by certain head-movements ${ }^{2}$

D) Stereotyped phenomenology in a particular patient ${ }^{5,6}$

E) Not better accounted for by another diagnosis.

\subsubsection{Notes}

1. The number of attacks was chosen because VP is an episodic vestibular disorder which usually presents with a high frequency of attacks. The frequency of attacks varies greatly among patients: from 30 attacks per day to a few attacks per year. The course of the disease is usually chronic (i.e., longer than three months) with some patients suffering hundreds of attacks per year.

2. The majority of attacks occur spontaneously ("out of the blue"). In some patients the attacks may be induced by turning the head to the right or left in the upright position. This seems to be similar to the induction of attacks in trigeminal neuralgia due to a sensory input. The triggering head or body movements typically do not have the same pattern as those in BPPV. In some of the patients, attacks of vertigo and nystagmus may be provoked by hyperventilation (5). If the attacks are reproducibly induced by a sustained lateral turn of the head one should consider rotational vertebral artery occlusion syndrome as a differential diagnosis. If the attacks are provoked by sudden intracranial pressure changes (such as might occur during sneezing, coughing or Valsalva maneuver) or by sudden changes in pressure in the ear canal or ambient atmosphere, perilymphatic fistula or an inner ear third mobile window syndrome such as superior canal dehiscence syndrome should be considered.

3. The type of vertigo (spinning or non-spinning) or directional pulsion is intra-individually quite uniform. If the attacks occur while standing or walking patients usually experience unsteadiness.

4. Duration: In most patients the attacks last from only a second to up to one minute; in other patients the duration of the attacks or some of the attacks may be longer up to many minutes or increase during the course of the disease.
Differential diagnoses with short attacks to consider are Tumarkin's otolith crisis, paroxysmal brainstem attacks, perilymph fistula and, rarely, epilepsy with vestibular aura (for "differential diagnosis" see below). In patients with longer-lasting attacks, other disorders, in particular vestibular migraine and Menière's disease should be considered.

5. Some patients may report auditory symptoms, such as unilateral tinnitus or hyperacusis during the attacks. Conclusions about the affected ear and the affected nerves can be drawn from the type of complaints - vestibular (originating from the canals or otolith organs) or cochlear symptoms [35]). If there is a combination of symptoms of other cranial nerves, the side of the lesion can possibly be deduced. Thus, for example, simultaneously occurring symptoms of the 7th and 8th cranial nerves (with vertigo, tinnitus and hemifacial spasm) [36] indicate an irritation of both cranial nerves in the internal acoustic meatus, where both lie in close proximity to each other.

6. If a patient is examined during an attack, a horizontal + torsional nystagmus time-locked to the attack and beating toward the affected ear may be observed.

7. The majority of patients respond to treatment with carbamazepine (200 to $800 \mathrm{mg}$ per day) or oxcarbazepine (300 to $900 \mathrm{mg}$ per day) [3, $5,10,14,39]$. Although yet not proven in a state-of-the-art randomized controlled clinical trial, response to a treatment with sodiumchannel blockers supports the diagnosis. This criterion is similar to the treatment response to indomethacin in paroxysmal hemicrania (International Headache Society Classification ICHD-II, 3.2). If a patient has not been treated yet this criterion cannot be applied and the diagnosis should be probable VP.

\subsection{Comments}

\subsubsection{Epidemiology}

Although the prevalence of VP is not known, it should be considered a rare disease $(<1$ in 2000 people), since only small case series and single cases have been published $[3,5,10,13,14,25,39]$. There are no data on the life-time prevalence of VP. The relative frequency of VP in a group of more than 17,000 patients with vertigo and dizziness in a tertiary care centre was around $4 \%$ [6]. The mean age 
of the patients in the three case series with more than 10 patients was 51 years (range 25-67 years) [5], $48.0 \pm 15.3$ years (mean \pm SD) [14] and $48.4 \pm 14.5$ years (range 25-77 years). Vestibular paroxysmia was also described in children with features similar to those in adults [25] and appears to have often a good long term prognosis with spontaneous remission with age [7]. From the three studies mentioned above $[6,14,25]$ of a total number of 63 patients, 32 were female. There is no epidemiological evidence of a genetic contribution.

\subsubsection{Pathophysiology and etiology}

In analogy to brief recurrent symptoms in trigeminal neuralgia, hemifacial spasm, glossopharyngeal neuralgia or myokymia of the superior oblique muscle [15], it is assumed that the short attacks of vertigo are triggered by ephaptic discharges, i.e. pathological paroxysmal interaxonal transmissions between neighboring, partially demyelinated axons. The likely site of the lesion is the central (oligodendroglia) myelin [12], proximal to the "transition zone". This corresponds to the first $15 \mathrm{~mm}$ after the nerve exit [21]. Potential causes for nerve injury are focal irritation by a blood vessel [2, 3, 5, 12, 16, 23, 32, 39], tumor or cyst $[1,24]$ compression, demyelination, trauma and unidentified causes.

\subsubsection{Laboratory examinations}

Approximately $50 \%$ of patients undergoing testing of vestibular and audiological function exhibit signs of a mild to moderate unilateral hypofunction during the attack-free intervals [14]. Hearing loss is usually less prominent than in patients with Menière's disease. Identification of the affected side is often not possible. If the attacks are accompanied by strictly unilateral audiological symptoms and there are vestibular and audiological deficits on the same side, one can identify the affected ear in exceptional cases [36].

\subsection{MR Imaging}

The role of MRI in identifying the affected side also has to be evaluated further [10, 14]. In a study of 32 patients with VP, neurovascular compression of the 8th cranial nerve was detected in $95 \%$ of the patients; bilateral neurovascular compression was found in $42 \%$ of the patients [14]. In another study of 20 patients with VP, neurovascular compression of the 8th cranial nerve was found in all patients, but also in 7 out of 20 control subjects (sensitivity: 100\%, specificity: 65\% for the diagnosis of VP by MRI) [3]. The distance between the brainstem and compressing vessels varied between 0.0 and $10.2 \mathrm{~mm}$. This part of the nerve is proximal to the transition zone and covered by oligodendrocytes. In 15 cases, the compressing vessel was the anterior inferior cerebellar artery $(75 \%)$, in one the posterior inferior cerebellar artery (5\%), in two a vein (10\%), and in another two the vertebral artery. Thus, a high-resolution MRI with CISS/FIESTA sequences of the brainstem may support the diagnosis.

Seven Tesla MRI was performed in six patients with VP [34]. The study confirmed neuro-vascular cross-compression also seen on 1.5 and 3.0 MRI. No structural abnormalities were detected in any of the patients in 7 Tesla MRI. These findings suggest that the symptoms of VP in these patients were not caused by structural nerve lesions.

In trigeminal neuralgia, high-resolution diffusion tensor imaging revealed significantly lower anisotropy and higher apparent diffusion coefficient in the affected trigeminal root compared to control, which correlated with structural atrophic nerve changes [22]. Comparable findings are not yet available for the 8th cranial nerve due to methodological limitations by the short course of the 8th cranial nerve from the brainstem to the internal acoustic meatus and the adjacent temporal bone. A cranial MRI should also be performed to exclude the presence of a tumor in the area of the cerebello-pontine angle, arachnoid cysts [1], megalodolichobasilaris, brainstem plaques in multiple sclerosis (MS), brainstem infarctions (leading to paroxysmal brainstem attacks with or without ataxia) or other brainstem lesions.

\subsection{Differential diagnosis}

The leading symptom of VP is recurrent spontaneous attacks of vertigo. The diagnosis is generally straightforward because of the characteristic brief duration (from seconds up to one minute), the frequently recurring attacks of vertigo and the response to a treatment with carbamazepine or oxcarbazepine. There are only a few other disorders which may present with this leading symptom:

- Menière's disease: duration of the attacks from 20 min to 12 hours, low- to medium-frequency sensorineural hearing loss $(>30 \mathrm{~dB},<2000 \mathrm{~Hz})$ [28].

- Tumarkin's otolithic crisis ("vestibular drop attacks"). These sudden falls are usually not 
accompanied by vertigo and occur most often in patients with known Menière's disease, typically while standing, whereas in VP the attacks occur in any body positions.

- Paroxysmal brainstem attacks with vertigo, dysarthria or ataxia (after stroke or in MS) may be difficult to distinguish, as they also respond to low doses of sodium-channel blockers. It was shown that they may be caused by a brainstem lesion due to MS plaques or lacunar infarctions [27], which also leads to ephaptic discharges of neighboring fibers of the brainstem paths. In such cases the use of MRI with thin brainstem slices is useful for establishing the diagnosis.

- Vestibular migraine [26]: officially the duration of the attacks is 5 min to 72 hours, current or previous history of migraine, most attacks being accompanied by other migrainous symptoms. In vestibular migraine, short spells of vertigo may be induced by changes of head or body position when patients are motion sensitive during an episode of vestibular migraine.

- Vertebrobasilar transient ischemic attacks: vertigo frequently occurs in isolation in this condition [33].

- Panic attacks: according to DSM-5, the diagnostic criteria for a panic attack include a discrete period of intense fear or discomfort, in which four (or more) of the following symptoms develop abruptly and reach a peak within minutes: feeling dizzy, unsteady, lightheaded, or faint; nausea or abdominal distress; palpitations, and/or accelerated heart rate; sweating; trembling or shaking; sensations of shortness of breath or being smothered; feeling of choking; chest pain or discomfort; de-realization or depersonalization; fear of losing control or going insane; sense of impending death; paresthesias; chills or hot flashes. Panic attacks are often longer than typical attacks of VP. It may be helpful to ask the patient which of the symptoms come first to differentiate between the two.

- Perilymph fistula: The cardinal symptoms of perilymph fistula (and superior canal dehiscence syndrome) are attacks of vertigo caused by changes in pressure, for example, by coughing, pressing, sneezing, lifting, or loud noises and accompanied by illusory movements of the environment (oscillopsia) and instability of posture and gait with or without hearing disorders. The attacks, which can last seconds to days, may also occur during changes in the position of the head (e.g., when bending over) and when experiencing significant changes in altitude (e.g., mountain tours, flights) [6].

- Episodic ataxia type 2: the duration of the attacks varies from several minutes to hours and more than $90 \%$ of the patients have cerebellar signs, in particular gaze-evoked nystagmus and downbeat nystagmus [20,40]. The onset of manifestations after the age of 20 is unusual. The much rarer episodic ataxia type 1 is another differential diagnosis. It is characterized by recurrent attacks of ataxia, dizziness and visual blurring, provoked by abrupt postural changes, emotion, vestibular stimulation and lasting minutes. These patients also have neuromyotonia, i.e. continuous spontaneous muscle fiber activity [19].

- Epilepsy with vestibular aura: Vestibular auras can manifest with short attacks of vertigo and nystagmus. Vestibular aura with additional symptoms, so-called non-isolated vestibular aura, is much more prevalent than isolated vestibular aura, which is rare. Vestibular aura is primarily associated with temporal lobe seizures. Isolated vestibular aura spells often last only a few seconds, but longer spells are also reported [41].

Other differential diagnoses are characterized by recurrent attacks of vertigo that are induced by certain maneuvers. These differential diagnoses include BPPV, central positional vertigo/nystagmus, "rotational vertebral artery occlusion syndrome" (RVAOS), orthostatic hypotension, or rarely cysts or tumors in the cerebello-pontine angle [1, 24]. In BPPV the attacks are induced by changes of head or body position relative to gravity, and the diagnosis can be proven by the diagnostic positional maneuvers. However, if they are negative, VP remains an important differential diagnosis. In central positional/positioning nystagmus the positioning maneuvers induce a similar nystagmus in different head positions [9]. In RVAOS the attacks are induced by rotation of the head either to the right or left, and diagnosis is proven by angiography. Similar to VP the symptoms are also caused by an excitation of the peripheral vestibular system [38]. In orthostatic hypotension the symptoms occur when the patient stands up and may be associated with vertigo and downbeat nystagmus; the key to this diagnosis is measurement of supine and orthostatic blood pressure [11]. 


\subsection{Therapy}

\subsubsection{Medical treatment}

A therapeutic trial of a low dose of carbamazepine (200-800 mg/day) or oxcarbazepine $(300-900 \mathrm{mg} /$ day) is often effective (for references see $[8,37])$. Moreover, a positive response supports the definite diagnosis. The exact specificity of the drug response for the establishment of the diagnosis still needs to be shown. A study on the course of the disease in 32 patients under treatment with carbamazepine or oxcarbazepine over a 3-year period revealed a significant and continuing decrease in the attack frequency down to $10 \%$ of the initial value as well as a reduction in the intensity and duration of the attacks [14]. In case of intolerance to these drugs, other sodium channel blockers such as phenytoin or valproic acid are possible alternatives; however, there are no study data available yet.

\subsubsection{Surgical treatment}

Despite the report of partial successes [31] and a clinically well-documented single case [39], operative microvascular decompression should be reserved for cases with VP who respond but do not tolerate the treatment with the above mentioned drugs and in whom the affected side could be clearly identified because of the risk of a brainstem infarction due to intra- or post-operative vasospasm.

\subsection{Areas of uncertainty}

- Epidemiological and clinical features of VP, including the nystagmus during the attacks, are not well known. Since there are no tests to prove the diagnosis of VP, further multicenter studies are needed to improve phenotyping, including familial history of VP.

- The role of imaging in the diagnosis and identification of the affected side is not clear because of the high rate of blood vessels close to the 8th cranial nerve in healthy subjects. Further, MRI with the appropriate sequences (CISS, FIESTA) to identify a neuro-vascular compression is not easily available in all countries.

- The role of therapy, i.e. the response to carbamazepine/oxcarbazepine, as a diagnostic criterion has to be further established, in particular, since there are no published randomized controlled trials on treatment.

\section{Acknowledgments}

This work was supported by the Federal Ministry of Education and Research to the German Center for Vertigo and Balance Disorders (Grant No. 01EO0901 and 01EO1401).

\section{References}

[1] V. Arbusow, M. Strupp, M. Dieterich, L. Jager, A. Hischa, P. Schulz and T. Brandt, Alternating episodes of vestibular nerve excitation and failure, Neurology 51 (1998), 1480-1483.

[2] R.A. Bertrand, P. Molina and J. Hardy, Vestibular syndrome and vascular anomaly in the cerebello-pontine angle, Acta Otolaryngol 83 (1977), 187-194.

[3] C. Best, J. Gawehn, H.H. Kramer, F. Thomke, T. Ibis, W. Muller-Forell and M. Dieterich, MRI and neurophysiology in vestibular paroxysmia: Contradiction and correlation, J Neurol Neurosurg Psychiatry 84 (2013), 1349-1356.

[4] A. Bisdorff, M. von Brevern, T. Lempert and D.E. NewmanToker, Classification of vestibular symptoms: Towards an international classification of vestibular disorders, $J$ Vestib Res 19 (2009), 1-13.

[5] T. Brandt and M. Dieterich, Vestibular paroxysmia: Vascular compression of the eighth nerve? Lancet 343 (1994), 798-799.

[6] T. Brandt, M. Dieterich and M. Strupp, Vertigo and dizziness - common complaints, Springer, London, 2013.

[7] T. Brandt, T. Huppert, K. Hufner, V.C. Zingler, M. Dieterich and M. Strupp, Long-term course and relapses of vestibular and balance disorders, Restor Neurol Neurosci 28 (2010), 69-82.

[8] T. Brandt, M. Strupp and M. Dieterich, Vestibular paroxysmia: A treatable neurovascular cross-compression syndrome, J Neurol 263(Suppl 1) (2016), 90-96.

[9] U. Buttner, C. Helmchen and T. Brandt, Diagnostic criteria for central versus peripheral positioning nystagmus and vertigo: A review, Acta Otolaryngol 119 (1999), 1-5.

[10] T.P. Chang, Y.C. Wu and Y.C. Hsu, Vestibular paroxysmia associated with paroxysmal pulsatile tinnitus: A case report and review of the literature, Acta Neurol Taiwan 22 (2013), 72-75.

[11] J.H. Choi, J.D. Seo, M.J. Kim, B.Y. Choi, Y.R. Choi, B.M. Cho, J.S. Kim and K.D. Choi, Vertigo and nystagmus in orthostatic hypotension, Eur J Neurol 22 (2015), 648-655.

[12] D. DeRidder, A. Moller, J. Verlooy, M. Cornelissen and L. DeRidder, Is the root entry/exit zone important in microvascular compression syndromes? Neurosurgery 51 (2002), 427-433.

[13] L.Y. He, W.W. Dong, W. Huang, Y. Luo and F.J. Lu, Clinical manifestations and imaging features of peripheral vestibular paroxysmia: A report of 7 cases, Zhonghua Yi Xue Za Zhi 89 (2009), 909-911.

[14] K. Hufner, D. Barresi, M. Glaser, J. Linn, C. Adrion, U. Mansmann, T. Brandt and M. Strupp, Vestibular paroxysmia: Diagnostic features and medical treatment, Neurology 71 (2008), 1006-1014.

[15] K. Hufner, J. Linn and M. Strupp, Recurrent attacks of vertigo with monocular oscillopsia, Neurology 71 (2008), 863. 
[16] P.J. Jannetta, Cranial nerve vascular compression syndromes (other than tic douloureux and hemifacial spasm), Clin Neurosurg 28 (1981), 445-456.

[17] P.J. Jannetta, Neurovascular cross-compression in patients with hyperactive dysfunction symptoms of the eighth cranial nerve, Surg Forum 26 (1975), 467-468.

[18] P.J. Jannetta, M.B. Moller and A.R. Moller, Disabling positional vertigo, N Engl J Med 310 (1984), 1700-1705.

[19] J.C. Jen, T.D. Graves, E.J. Hess, M.G. Hanna, R.C. Griggs and R.W. Baloh, Primary episodic ataxias: Diagnosis, pathogenesis and treatment, Brain 130 (2007), 2484-2493.

[20] J.C. Jen, G.W. Kim and R.W. Baloh, Clinical spectrum of episodic ataxia type 2, Neurology 62 (2004), 17-22.

[21] J. Lang, Anatomy, length and blood vessel relations of "central" and "peripheral" paths of intracisternal cranial nerves, Zentralbl Neurochir 43 (1982), 217-258.

[22] P.R. Leal, J.A. Roch, M. Hermier, M.A. Souza, G. CristinoFilho and M. Sindou, Structural abnormalities of the trigeminal root revealed by diffusion tensor imaging in patients with trigeminal neuralgia caused by neurovascular compression: A prospective, double-blind, controlled study, Pain 152 (2011), 2357-2364.

[23] T.A. Leclercq, C.L. Hill and F. Grisoli, Retromastoid microsurgical approach to vascular compression of the eighth cranial nerve, Laryngoscope 90 (1980), 1011-1017.

[24] S.U. Lee, S.H. Jeong, H.J. Kim and J.S. Kim, Cerebellopontine angle meningioma mimicking vestibular paroxysmia, J Neurol 263 (2016), 168-170.

[25] N. Lehnen, T. Langhagen, F. Heinen, D. Huppert, T. Brandt and K. Jahn, Vestibular paroxysmia in children: A treatable cause of short vertigo attacks, Dev Med Child Neurol 57 (2015), 393-396.

[26] T. Lempert, J. Olesen, J. Furman, J. Waterston, B. Seemungal, J. Carey, A. Bisdorff, M. Versino, S. Evers and D. Newman-Toker, Vestibular migraine: Diagnostic criteria, J Vestib Res 22 (2012), 167-172.

[27] Y. Li, C. Zeng and T. Luo, Paroxysmal dysarthria and ataxia in multiple sclerosis and corresponding magnetic resonance imaging findings, J Neurol 258 (2011), 273-276.

[28] J.A. Lopez-Escamez, J. Carey, W.H. Chung, J.A. Goebel, M. Magnusson, M. Mandala, D.E. Newman-Toker, M. Strupp, M. Suzuki, F. Trabalzini and A. Bisdorff, Diagnostic criteria for Meniere's disease, $J$ Vestib Res 25 (2015), 1-7.

[29] M.B. Moller and A.R. Moller, Vascular compression syndrome of the eighth nerve. Clinical correlations and surgical findings, Neurol Clin 8 (1990), 421-439.

[30] M.B. Moller, A.R. Moller, P.J. Jannetta, H.D. Jho and L.N. Sekhar, Microvascular decompression of the eighth nerve in patients with disabling positional vertigo: Selection criteria and operative results in 207 patients, Acta Neurochir (Wien) 125 (1993), 75-82.

[31] M.B. Moller, A.R. Moller, P.J. Jannetta and L. Sekhar, Diagnosis and surgical treatment of disabling positional vertigo, J Neurosurg 64 (1986), 21-28.

[32] B.F. McCabe and B.J. Gantz, Vascular loop as a cause of incapacitating dizziness, Am J Otol 10 (1989), 117-120.

[33] N.L. Paul, M. Simoni and P.M. Rothwell, Transient isolated brainstem symptoms preceding posterior circulation stroke: A population-based study, Lancet Neurol 12 (2013), 65-71.

[34] P.S. Rommer, G. Wiest, C. Kronnerwetter, H. Zach, B. Loader, K. Elwischger and S. Trattnig, 7-Tesla MRI demonstrates absence of structural lesions in patients with vestibular paroxysmia, Front Neuroanat 9 (2015), 81.

[35] D. Russell and R.W. Baloh, Gabapentin responsive audiovestibular paroxysmia, J Neurol Sci 281 (2009), 99-100.

[36] A. Straube, U. Buttner and T. Brandt, Recurrent attacks with skew deviation, torsional nystagmus, and contraction of the left frontalis muscle, Neurology 44 (1994), 177-178.

[37] M. Strupp, M. Dieterich, T. Brandt and K. Feil, Therapy of Vestibular Paroxysmia, Superior Oblique Myokymia, and Ocular Neuromyotonia, Curr Treat Options Neurol 18 (2016), 34.

[38] M. Strupp, J.H. Planck, V. Arbusow, H.J. Steiger, H. Bruckmann and T. Brandt, Rotational vertebral artery occlusion syndrome with vertigo due to "labyrinthine excitation", Neurology 54 (2000), 1376-1379.

[39] M. Strupp, S. von Stuckrad-Barre, T. Brandt and J.C. Tonn, Teaching NeuroImages: Compression of the eighth cranial nerve causes vestibular paroxysmia, Neurology 80 (2013), e77.

[40] M. Strupp, A. Zwergal and T. Brandt, Episodic ataxia type 2, Neurotherapeutics 4 (2007), 267-273.

[41] A.A. Tarnutzer, S.H. Lee, K.A. Robinson, P.W. Kaplan and D.E. Newman-Toker, Clinical and electrographic findings in epileptic vertigo and dizziness: A systematic review, $\mathrm{Neu}$ rology 84 (2015), 1595-1604.

[42] M. von Brevern, P. Bertholon, T. Brandt, T. Fife, T. Imai, D. Nuti and D. Newman-Toker, Benign paroxysmal positional vertigo: Diagnostic criteria, J Vestib Res 25 (2015), 105-117.

[43] L. Yap, V.B. Pothula and T. Lesser, Microvascular decompression of cochleovestibular nerve, Eur Arch Otorhinolaryngol 265 (2008), 861-869. 